\title{
Early postoperative imaging after non-bariatric gastric resection: a primer for radiologists
}

\author{
Massimo Tonolini ${ }^{\cdot}$ Elena Bracchi $^{1}$
}

Received: 10 April 2017 /Revised: 2 May 2017 / Accepted: 4 May 2017 / Published online: 19 June 2017

(C) The Author(s) 2017. This article is an open access publication

\begin{abstract}
Surgical resection represents the mainstay treatment and only potentially curative option for gastric carcinoma, and is increasingly performed laparoscopically. Furthermore, other tumours and selected cases of nonmalignant disorders of the stomach may require partial or total gastrectomy. Often performed in elderly patients, gastric resection remains a challenging procedure, with significant morbidity (14-43\% complication rate) and non-negligible postoperative mortality (approximately $3 \%$ ). This paper provides an overview of contemporary surgical techniques for non-bariatric gastric resection, reviews and illustrates the expected postoperative imaging appearances, common and unusual complications after partial and total gastrectomy. Albeit cumbersome or unfeasible in severely ill or uncooperative patients, contrast fluoroscopy remains useful to rapidly check for anastomotic patency and integrity. Currently, emphasis is placed on multidetector CT, which comprehensively visualizes the surgically altered anatomy and consistently detects complications such as anastomotic leaks and fistulas, duodenal stump leakage, afferent loop syndrome, haemorrhages, pancreatic fistulas and portomesenteric venous thrombosis. Our aim is to help radiologists become familiar with early postoperative imaging, in order to understand the surgically altered anatomy and to differentiate between expected imaging
\end{abstract}

Massimo Tonolini

mtonolini@sirm.org

1 Department of Radiology, "Luigi Sacco" University Hospital, Via G.B. Grassi 74, 20157 Milan, Italy appearances and abnormal changes heralding iatrogenic complications, thus providing a consistent basis for correct choice between conservative, interventional or surgical treatment.

\section{Teaching points}

- Radical gastrectomy is associated with frequent postoperative morbidity and non-negligible mortality.

- In cooperative patients fluoroscopy allows checking for anastomotic patency and leaks.

- Multidetector CT with / without oral contrast comprehensively visualizes the operated abdomen.

- Awareness of surgically altered anatomy and expected postoperative appearances is warranted.

- Main complications include anastomotic and duodenal leaks, haemorrhages and pancreatic fistulas.

Keywords Gastrectomy · Complications · Anastomotic leakage $\cdot$ Contrast fluoroscopy $\cdot$ Computed tomography (CT)

\section{Introduction}

Worldwide, gastric carcinoma (GC) represents one of the leading cancers and accounts for over 900,000 new cases and 723,000 deaths yearly [1]. GC shows dramatic geographic variation, with the highest incidence in China and Japan, parts of South America, Eastern Europe and Russia. Conversely, in North America and Europe this lethal disease has significantly declined because of improved nutrition, food refrigeration, eradication of Helicobacter pylori infection, decreasing tobacco use and alcohol intake. Whereas in the far East early GC detection through screening programs generally allows curative treatment, in the rest of the world most 

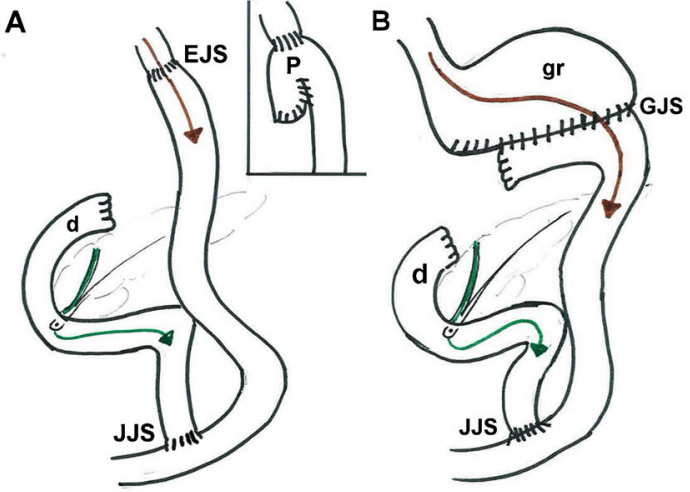

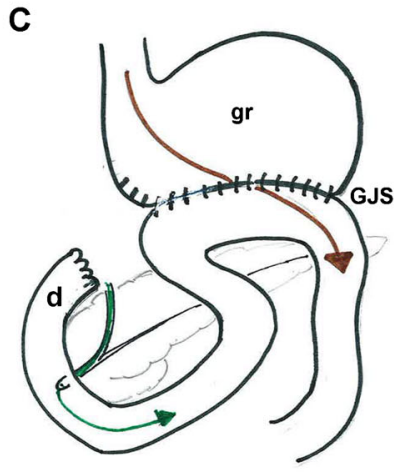

Fig. 1 Schematic representations of post-surgical anatomy: A) after total gastrectomy with Roux-en-Y esophago-jejunostomy (EJS), jejunojejunostomy (JJS) and blind-ending duodenal stump (d); alternative technique with pouch $(\mathrm{P})$ creation. Alimentary flow indicated by brown arrow, bile flow in dark green. B) after subtotal gastrectomy with smallsized gastric remnant (gr) connected to Roux-en-Y gastrojejunostomy (GJS). C) after partial gastrectomy reconstructed with a Billroth II GJS
GCs are diagnosed at an advanced stage, resulting in dismal prognosis (27\% 5-year survival rate) [1].

Resection with negative surgical margins represents the mainstay treatment and only potentially curative option for localized GCs, combined with adjuvant chemotherapy or chemo-radiotherapy for disease stages IB and higher. Radical gastrectomy remains a challenging surgical procedure with significant postoperative morbidity (14-43\% complication rate) and mortality $(0.8-12 \%$, mean 3\%) [2-6].

Traditionally, early post- gastrectomy imaging was limited to contrast fluoroscopy (CF) to assess anastomotic patency and integrity. More recently, multidetector CT is increasingly adopted to investigate most postoperative abdominal conditions, to comprehensively visualize the surgically altered anatomy and detect iatrogenic complications. However, compared to the literature about bariatric surgery imaging [7-9], few reports describe CT techniques and expected and abnormal appearances shortly after radical gastrectomy [10-12].

This paper provides an overview of contemporary surgical techniques, reviews and illustrates the expected postoperative imaging findings and complications after partial and total gastrectomy, aiming to provide radiologists with an increased familiarity in the interpretation of early post-gastrectomy CT studies and, ultimately, to limit iatrogenic morbidity.

\section{Overview of gastrectomy techniques}

Surgical exploration and aggressive resection of GC with curative intent is undertaken unless medical contraindications exist or preoperative imaging shows metastatic spread or major vascular invasion. Surgical approaches depend on tumour site and extension: tumours of the cardia, proximal and mid-body stomach require total gastrectomy with removal of the entire stomach along with terminal oesophagus and proximal duodenum. Conversely, GCs of the distal body and antrum may be treated by partial gastrectomy, with stapling
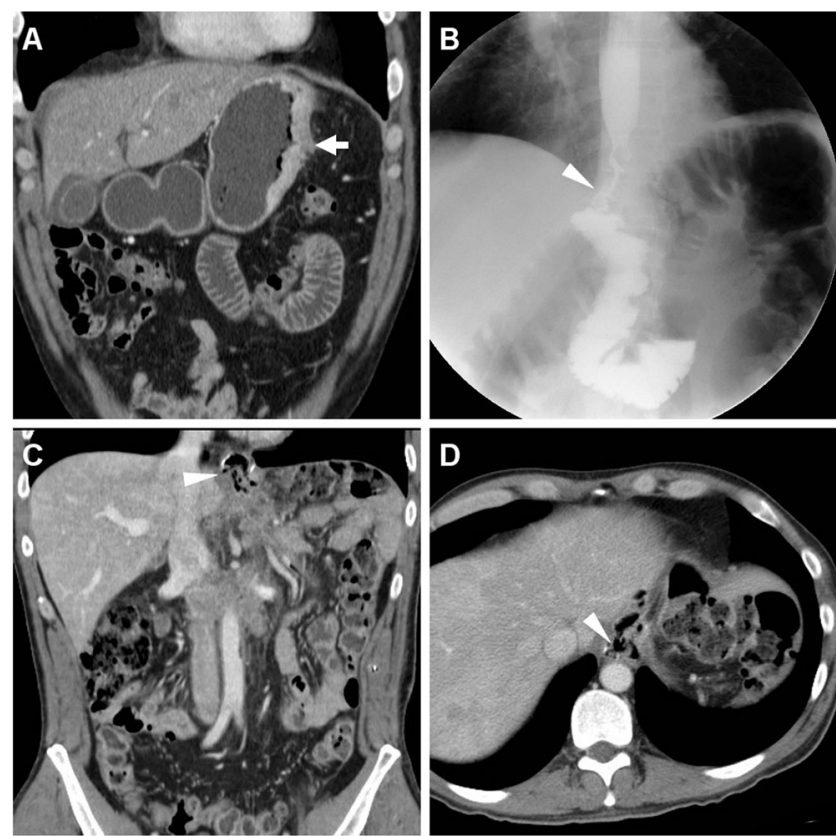

Fig. 2 In a 39-year-old male with endoscopic and bioptic diagnosis of stomach adenocarcinoma, preoperative staging $\mathrm{CT}$ with oral water distension (A) showed marked solid, enhancing mural thickening from the gastric fundus along the greater curvature (thick arrow). On the fifth postoperative day (POD) after uncomplicated total gastrectomy with lymphadenectomy and splenectomy, contrast fluoroscopy (CF, B) showed normally patent, thin EJS (arrowhead) with opacification of jejunal limb and no anastomotic contrast medium leakage. Corresponding expected postsurgical contrast-enhanced CT $(C, D)$ appearances at hospital discharge included stapled EJS (arrowheads) without peri-anastomotic air or fluid, absent spleen without collections in the surgical site 
and division of the stomach at least $6 \mathrm{~cm}$ from any macroscopic tumour [2-5, 13].

Radical gastrectomy is completed with splenectomy and partial pancreatectomy in $17.5 \%$ and $7.1 \%$ of patients, respectively. The extent of lymphadenectomy remains controversial: compared to standard nodal dissection of perigastric nodes along the lesser and greater curvature, D2 lymphadenectomy is extended along the celiac axis, left gastric, common hepatic and splenic arteries. Gastric resection is sometimes performed to treat uncommon stomach malignancies (gastrointestinal stromal tumours, lymphomas or sarcomas) and selected benign conditions such as refractory peptic ulcers. Very recently, oncologic gastric surgery has also been performed using laparoscopy, resulting in decreased intraoperative blood loss and shorter hospital stays [2-5, 14, 15].

Following gastrectomy, the choice of digestive tract anatomic reconstruction depends on the extent of resection. Most total gastrectomies are reconstructed with Roux-en-Y esophagojejunostomy (EJS), in which a jejunal limb is brought up and re-anastomosed to the distal oesophagus and to the closed duodenal stump (DS) (Fig. 1a). Whereas in
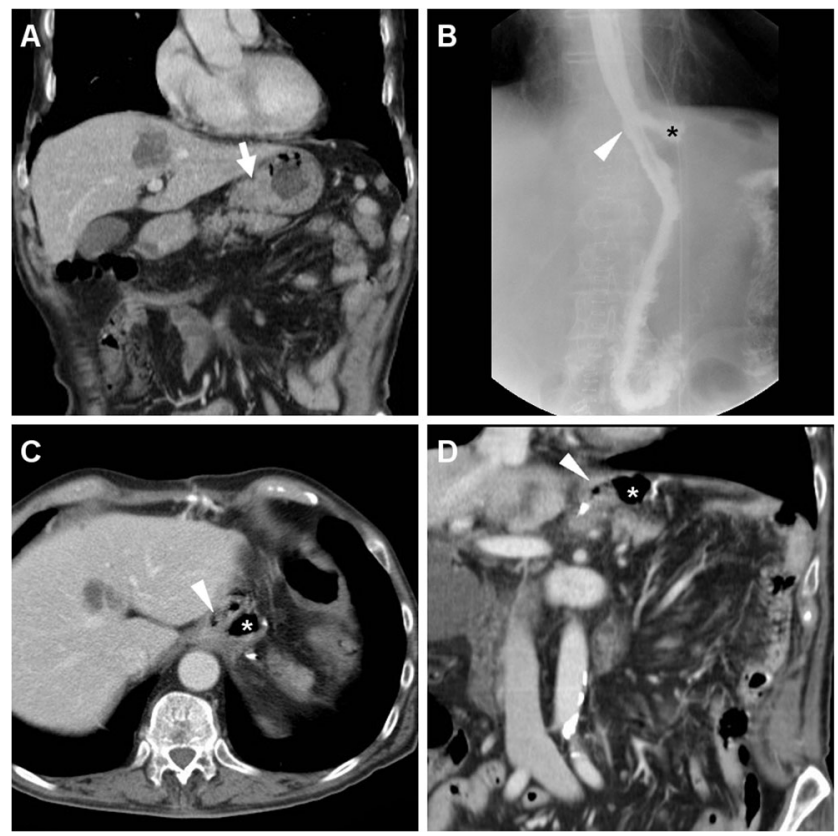

Fig. 3 In a 74-year-old male, preoperative CT (A) depicted a stricturing carcinoma of the gastric body (thick arrow). Six days after uncomplicated total gastrectomy with lymphadenectomy and splenectomy, CF (B) visualized normally patent EJS (arrowhead) with a seemingly extraluminal contrast medium accumulation (*) which corresponded to anastomotic recess of the Roux-en-Y EJS at surgical correlation, a finding which potentially mimics leakage if technical details are not known. Corresponding expected postsurgical CT changes including air-filled anastomotic recess $(*)$, non-stapled EJS (arrowheads) with unremarkable perianastomotic fat planes, no abnormal collections in the splenectomy site
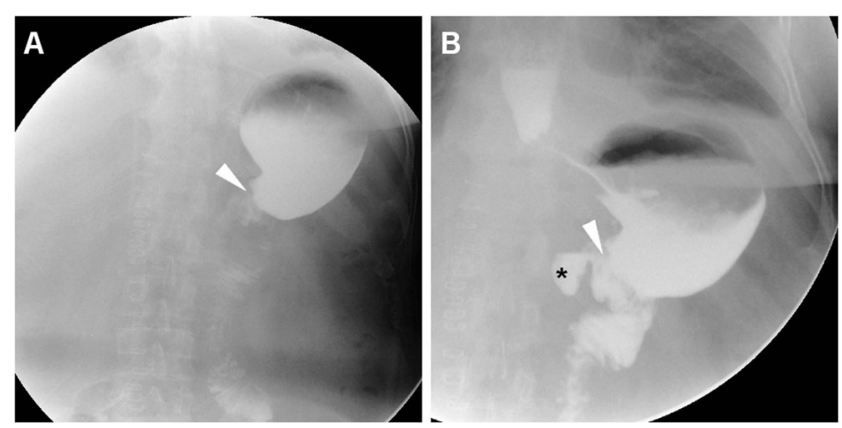

Fig. 4 In a 62-year-old male, CF performed five days after uncomplicated partial gastrectomy showed opacified, moderately dilated remnant stomach, slow transit of iodinated contrast through the GJS (arrowheads) and opacified Roux-en-Y anastomotic recess (*)

"high" subtotal gastrectomy the small-sized gastric remnant is connected to a Roux-en-Y gastrojejunostomy (GJS) (Fig. 1b), "low" partial gastrectomy with preservation of the gastric fundus may be reconstructed using a Billroth II GJS (Fig. 1c) technique, with DS directly connected to the remnant stomach. According to the surgeon's preference, anastomoses may be either hand-sewn or stapled $[5,13,16]$.
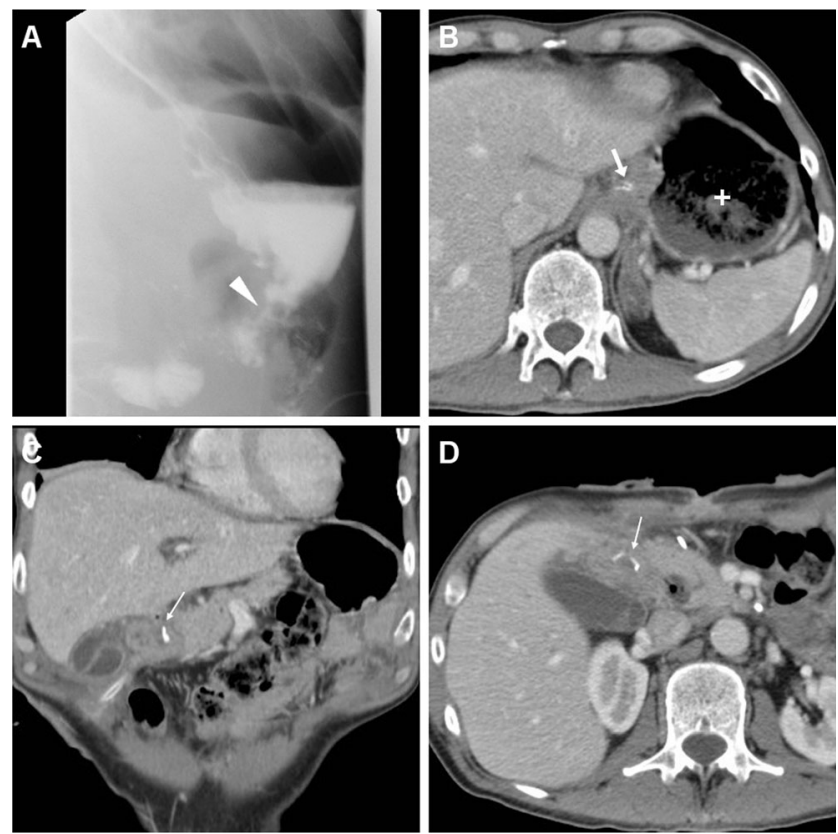

Fig. 5 A 63-year-old male underwent partial gastrectomy with Billroth II reconstruction to relieve a large, non-malignant pyloric ulcer refractory to medical therapy. Early postsurgical CF (A) showed mildly delayed transit of iodinated contrast through the GJS (arrowhead), with fluid level in the gastric remnant. Corresponding CT (B-D) appearances before discharge included dilated remnant stomach (+) with ingested material, surgical staples at the gastric resection site (arrow in B), expected imaging appearance of the duodenal stump (DS, thin arrows in C, D) indicated by a single metallic staple, without surrounding fluid or collections 

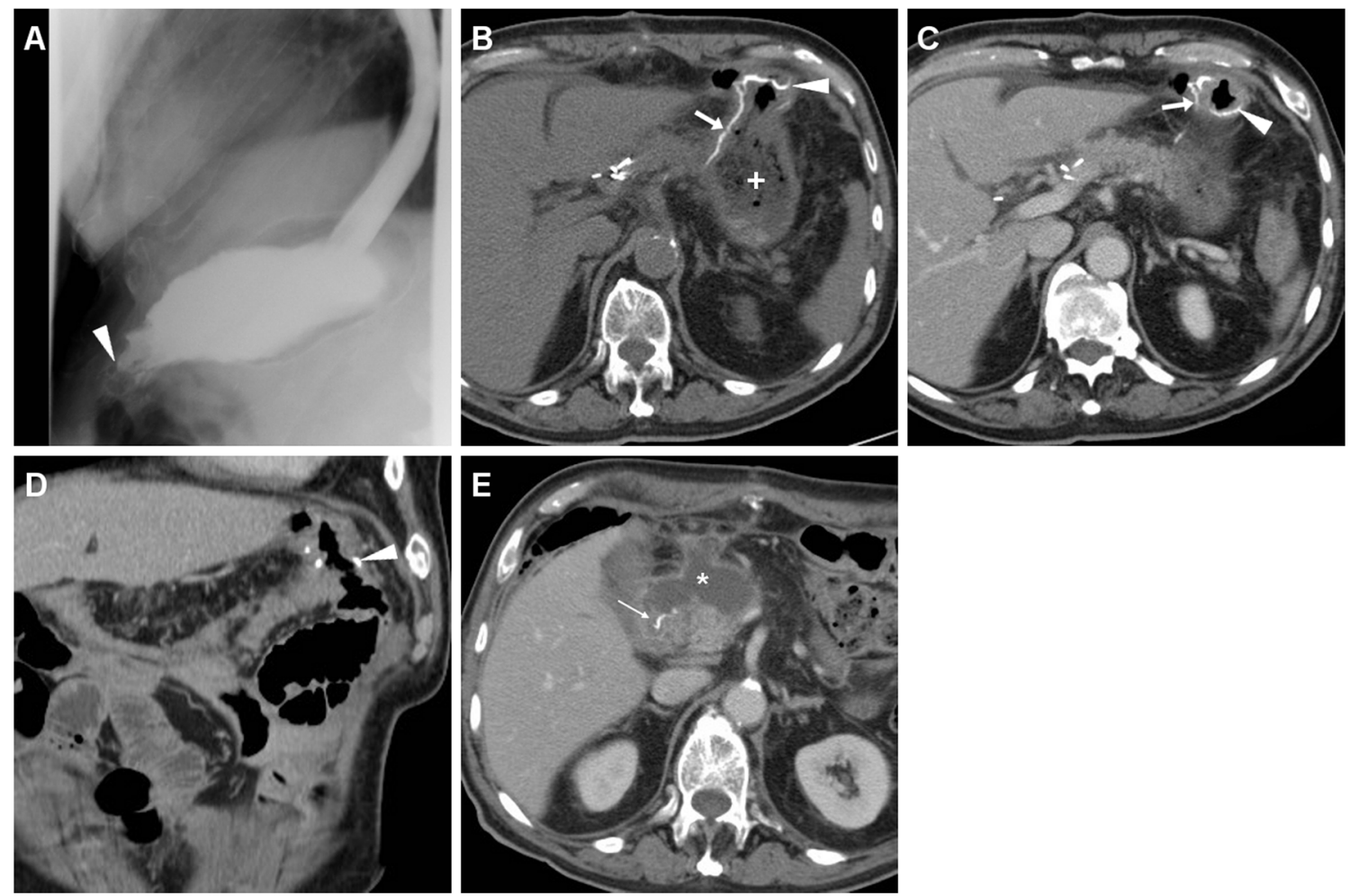

Fig. 6 A 78-year-old male with antral gastric cancer underwent subtotal gastrectomy with D2 lymphadenectomy and Billroth II reconstruction and suffered from postoperative vomiting and fever. On 5th POD CF (A) showed limited, slow transit through the GJS (arrowhead). On 9th POD, unenhanced (B) and post-contrast (C-E) CT showed stapled gastric resection site (arrows) and GJS (arrowheads), minimally dilated remnant stomach (+), and a fluid collection (* in E) abutting the DS (thin arrow) consistent with DS leakage, which was successfully managed conservatively but required prolonged hospitalization

\section{What to expect after radical gastrectomy}

The likelihood of postoperative morbidity is strongly influenced by extended operation time, which acts as a surrogate of challenging surgical manoeuvres. No significant differences in complication rates exist with regard to resection extension between total, proximal or distal gastrectomy. Compared to open surgery, laparoscopic and laparoscopic-assisted techniques are not associated with significantly different morbidity, particularly concerning pulmonary complications, anastomotic leakage and intra-abdominal abscesses. Conversely, splenectomy and pancreatectomy represent risk factors for additional morbidity $[2-5,14,15]$. The effect of extended lymphadenectomy remains controversial: several studies showed that D2 nodal dissection is associated with higher postoperative morbidity, reoperation rates and mortality, specifically with increased likelihood of pulmonary complications, wound infection, failed anastomosis and pancreatic disorders [17, 18].

Similarly to other major abdominal surgeries, respiratory problems including pleural effusion, atelectasis and pneumonia commonly occur after oncologic gastrectomy, particularly in elderly men with chronic obstructive lung disease $[4,19]$.

The most important specific post-gastrectomy complications include anastomotic and DS leakage, pancreatic fistula, acute pancreatitis and intra-abdominal haemorrhage, and ultimately require reintervention in $25 \%$ of cases, particularly because of leakage, abscesses and bleeding. Anastomotic complications are most feared and account for most of septic morbidity and mortality $[2-6,20]$.

The commonest manifestations of intra-abdominal complications include physical and laboratory (leukocytosis, increasing C-reactive protein levels) signs of sepsis within the first 7 to 10 postoperative days (PODs). Bleeding is generally heralded by hypotension, dropping haematocrit, blood from nasogastric or drainage tubes. Most surgeons increasingly feel that abdominal pain and physical findings are relatively insensitive and increasingly rely on early postoperative imaging. When discussing indication and timing of post-surgical studies, asking the surgeon to draw the reconstructed anatomy generally proves helpful to interpret fluoroscopic and CT appearances [4, 21]. 

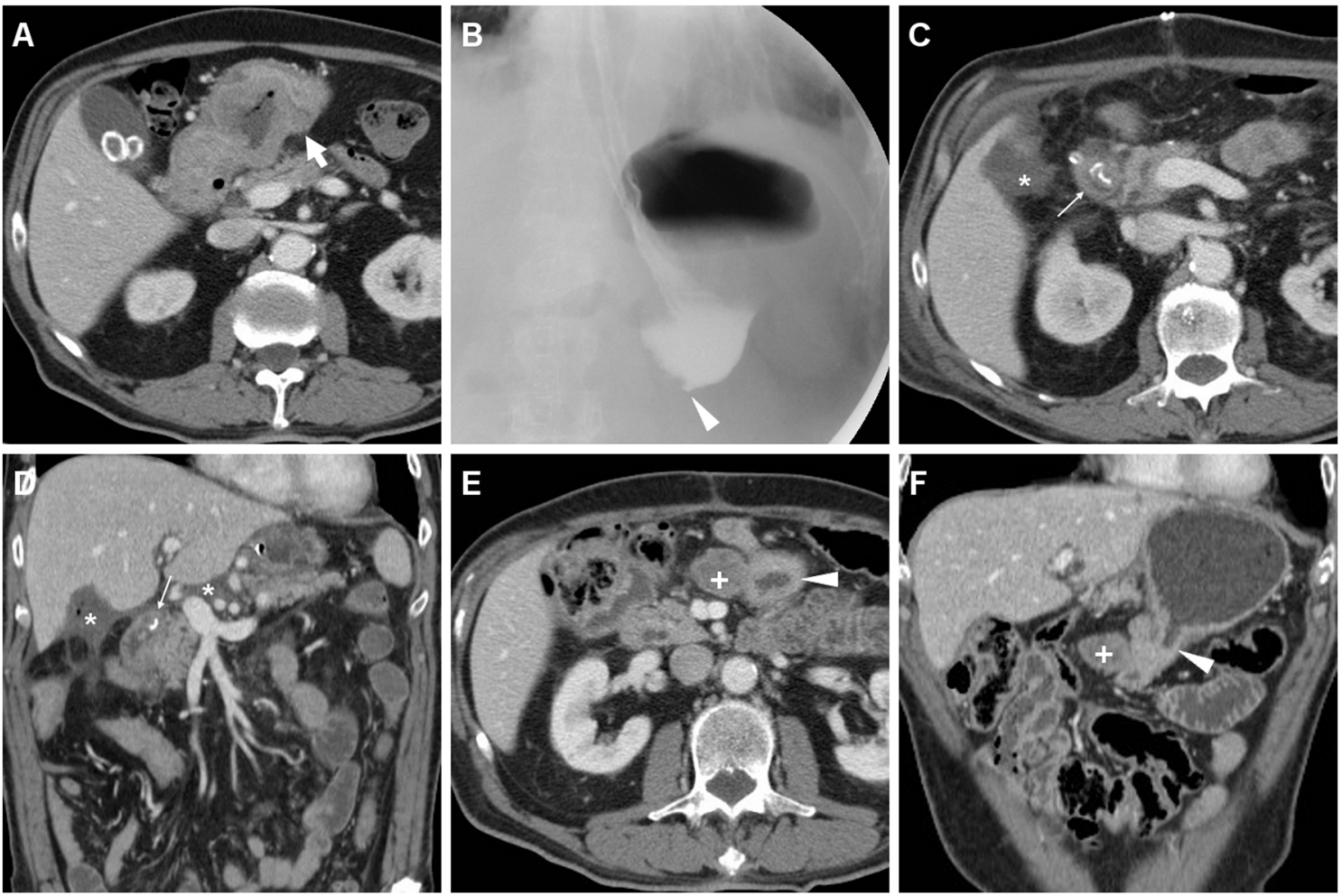

Fig. 7 A 76-year-old male with stricturing antro-pyloric carcinoma (thick arrow) as preoperatively depicted by CT (A) underwent subtotal gastrectomy with Roux-en-Y reconstruction. Early postoperative CF (B) showed little or no transit through the GJS (arrowhead). On 8th POD during postoperative sepsis, contrast-enhanced CT (C, D) showed normal stapled DS (thin arrows),

minimal fluid $(*)$ in the cholecystectomy site. The patient then improved on conservative therapy including gastric intubation. Subsequent endoscopy showed ischaemic ulceration of the GJS. Repeated CT (E, F) three months later showed circumferential mural thickening of the GJS (arrowheads) and perianastomotic nodule $(+)$ consistent with tumour recurrence
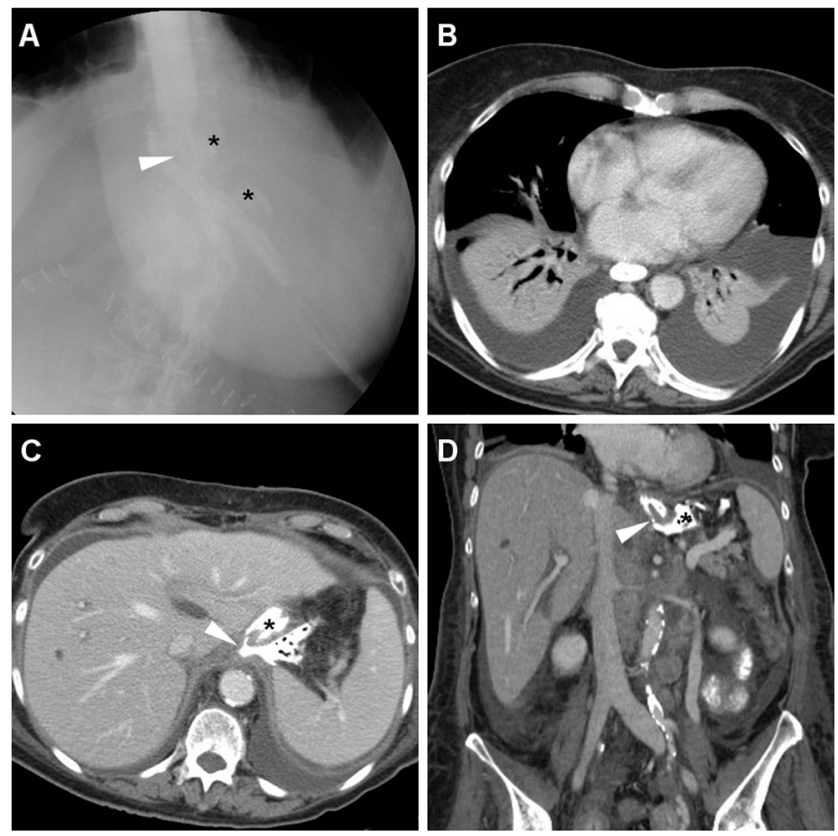

Fig. 8 In a 69-year-old woman with advanced carcinoma of the gastric body, palliative total gastrectomy was performed. Five days after surgery, CF (A) showed patent EJS with opacification of extensive anastomotic leakage (*). A few days later, on conservative management, contrastenhanced CT (B-D) showed bilateral pleural effusions and atelectasis (B), patent EJS (arrowheads) and persistent leak (*)

\section{Early post-gastrectomy imaging: techniques and normal appearances}

\section{Contrast fluoroscopy}

Fluoroscopic examination after oral administration of watersoluble CM has been largely adopted to check for possible anastomotic leakage. Albeit several institutions routinely perform CF within five PODs, testing of anastomosis should currently be obviated in asymptomatic patients [22, 23]. According to radiologist's preference, either low-osmolar iodinated CM or diatrizoate meglumine may be used, but the latter is hyperosmolar and may induce pulmonary oedema if aspirated $[24,25]$.

Rapid sequences in frontal and oblique views are acquired during ingestion or sipping of $\mathrm{CM}$ via a straw, with the patient standing or sitting on the fluoroscopy table: unfortunately, CF is very cumbersome when the recently operated patient is unable to cooperate or cannot swallow [24]. Compared to the EJS, which is generally recognized at the level or immediately below the diaphragmatic hiatus (Figs. 2 and 3), the position of a GJS is more variable and requires higher degrees of left- or right-sided patient rotation (Figs. 4, 5 and 6). 
Table 1 Checklist for interpretation of early post-gastrectomy CT

Feature Comments

Report pleuropulmonary changes at lung bases

Quantify free intraperitoneal air and/or fluid

Identify stapled or hand-sewn

- esophagojejunostomy (EJS) after total gastrectomy (Figs. 2 and 3) Eff

- gastrojejunostomy (GJS) after partial gastrectomy (Figs. 5 and 6)

- look for localized air, fluid or haemorrhagic collections

adjacent to either EJS or GJS (Figs.11 and 12)

Identify the closed duodenal stump (DS)

- assess dilatation (Fig. 15)

- look for adjacent collections (Figs. 6 and 9)

Report presence and site of drainage tubes

Assess post-splenectomy or post-pancreatectomy status

Assess patency of splenic, portal and mesenteric veins

Exclude retained foreign bodies

Scrutinize laparotomy site
Atelectasis, pneumonia, pleural effusion (see Fig. 8) or empyema (Fig. 14)

Discussion in text; see Fig. 10

Gastric remnant generally indicated by staple line

Efferent jejunal limb from EJS or GJS characterized by valvulae conniventes and tubular configuration on coronal viewing

DS generally recognized by a metallic staple at its blind end (Figs. 5, 6, 7 and 9)

If performed (see Figs. 2, 3 and 11)

For postoperative thrombosis, favoured by intra-abdominal sepsis

E.g. surgical sponges (indicated by thin hyperattenuating structures), bioabsorbable haemostatic materials agents such as Gelfoam or Surgicel (which appear as walled heterogeneous masses with internal "mottled" gas bubbles)

For abscess collections suggesting wound infection
After uncomplicated partial gastrectomy, delayed CM passage through the GJS, incomplete emptying and fluid-fluid level in the gastric remnant are fairly common, most usually self-limiting and secondary to ileus or anastomotic oedema, and generally do not represent a complication (Fig. 4); however, in our experience anastomotic ulceration may have a
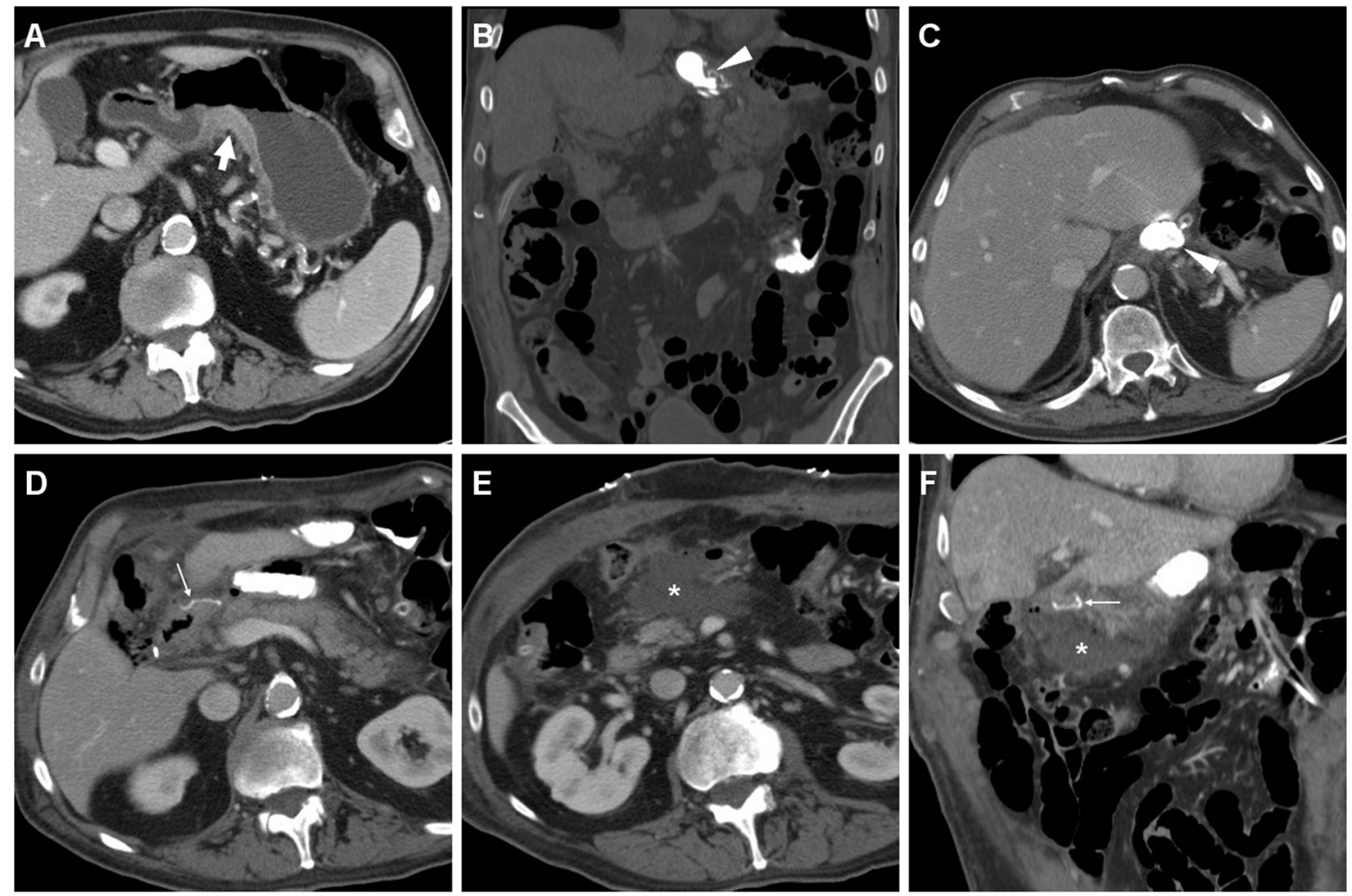

Fig. 9 An 81-year-old male with T3 N2 cancer of the lesser gastric curvature (thick arrow) as preoperatively depicted by $\mathrm{CT}$ with oral water distension (A) underwent total gastrectomy with Roux-en-Y reconstruction. On 9th POD CT was performed because of bile from drainage: unenhanced (B) and post contrast (C-F) scans with oral contrast showed normally opacified and patent

EJS (arrowheads) without evidence of anastomotic leak (note tip of surgical drainage abutting the EJS in C). Abutting the stapled DS (thin arrows) a non-capsulated fluid collection (*) corresponded to DS leakage which was conservatively managed 
similar appearance and may ultimately result in stricture (Fig. 7) [5].

Albeit perianastomotic extravasation (Fig. 8) is nearly $100 \%$ specific for leakage, a pitfall of CF is represented by misinterpretation of the anastomotic recess of an endto-side reconstruction as extraluminal CM (Figs. 3 and 4) [12].

\section{Early post-gastrectomy CT}

Nowadays, in most post-surgical situations, multidetector CT rapidly and consistently provides panoramic visualization of postoperative abdominal changes, and generally adds crucial information for diagnosis of iatrogenic complications [10,11,26].

Due to high prevalence of pleuropulmonary changes (Fig. 8), the CT acquisition should encompass the entire abdomen and lung bases. Some authors reported that early post-gastrectomy $\mathrm{CT}$ without oral $\mathrm{CM}$ is easier, faster and safer to perform shortly, particularly in bed-ridden uncooperative patients. Precontrast scans and lung or viewing with bone window settings easily allow identifying metallic surgical staples and intraperitoneal air, and differentiating between fluid and haemorrhagic collections. Unless contraindicated by allergy or renal failure, $\mathrm{CM}$-enhanced acquisition, at least in the portal-venous phase, is warranted. When clinical or laboratory findings suggest possible postoperative bleeding, an additional arterial-phase scanning may allow detection of active haemorrhage. In our experience, CT review along axial, coronal and sagittal planes may be beneficial.

A useful checklist for CT interpretation is provided in Table 1. Postoperative pneumoperitoneum is a common, expected finding which typically distributes freely and resolves within a few days. On plain radiographs or CT, intraperitoneal air may last up to 9-10 PODs, but decreases on serial imaging. Conversely, persisting or increasing gas raises concern for anastomotic dehiscence or visceral perforation. Following laparoscopy, the amount of expected intraperitoneal air is less compared to open surgery, because insufflated $\mathrm{CO} 2$ is rapidly absorbed; conversely, subcutaneous emphysema may result from insufflation in the abdominal wall [26, 27].

Borrowing from experience in bariatric surgery, oral administration of positive water-soluble CM may improve the CT performance, particularly regarding anastomotic leaks. If the patient can stand and swallow without risk of aspiration, we instruct him or her to drink low-osmolar $\mathrm{CM}$ such as $3 \%$ diluted iopamidol or iohexol 5-10 min prior to CT. Lack of extraluminal CM is strongly consistent with anastomosis integrity (Fig. 9). Finally, CT results strongly influence reoperation rate, mortality, duration of postoperative fasting and hospitalization [10-12].

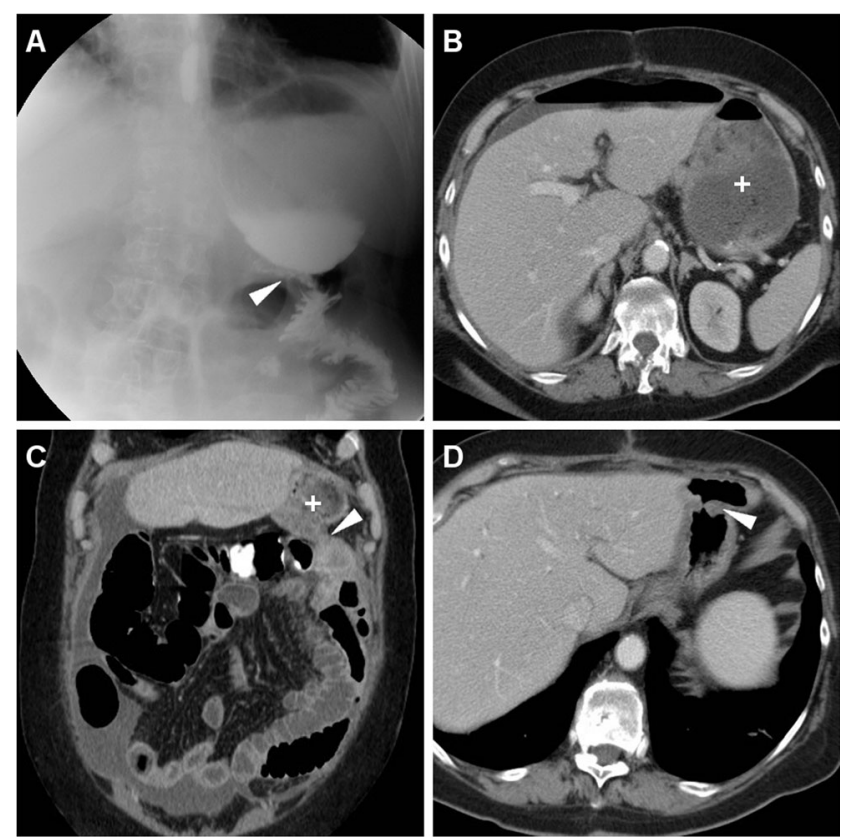

Fig. 10 A 77-year-old female with distal gastric carcinoma underwent partial gastrectomy. Early postoperative CF (A) showed slow emptying of gastric remnant through the GJS (arrowhead), without appreciable extraluminal leaks. Six days later, the patient suffered from high fever: contrast-enhanced CT (B-C) showed dilated remnant stomach (+), absence of collections adjacent to the GJS (arrowheads in C and D), but persistent intraperitoneal air and fluid consistent with peritonitis, confirmed at surgical exploration: after redo anastomosis, the final status of GJS (arrowhead) is shown in D

\section{Anastomotic complications}

\section{Anastomotic leaks}

Leakage resulting from breakdown of a suture line secondary to either inadequate technique, excessive tension or ischaemia, occurs after $3-4 \%$ of gastrectomies with either hand-sewn and stapled anastomosis, generally as an early complication within the first PODs; Incidence of leakage may reach $21 \%$ with routine use of CT. Albeit variably symptomatic, anastomotic dehiscence represents an independent predictor of survival with $10-20 \%$ associated mortality [2-4].

The fluoroscopic hallmark of anastomotic leak is represented by CM extravasation adjacent to a GJS or EJS (Fig. 8), with either contained collection or free dispersal in the adjacent surgical cavity. However, since the reported sensitivity varies in the range $22 \%$ to $67 \%$, negative $\mathrm{CF}$ findings do not rule out the possibility of leakage (Fig. 10) [22].

As discussed above, the use of $\mathrm{CT}$ is favoured over $\mathrm{CF}$ in uncooperative patients. Even without oral CM, CT is felt to be superior to $\mathrm{CF}$ with $89.5 \%$ positive predictive value compared to $40 \%$, mostly because CT shows both the anastomosis and 

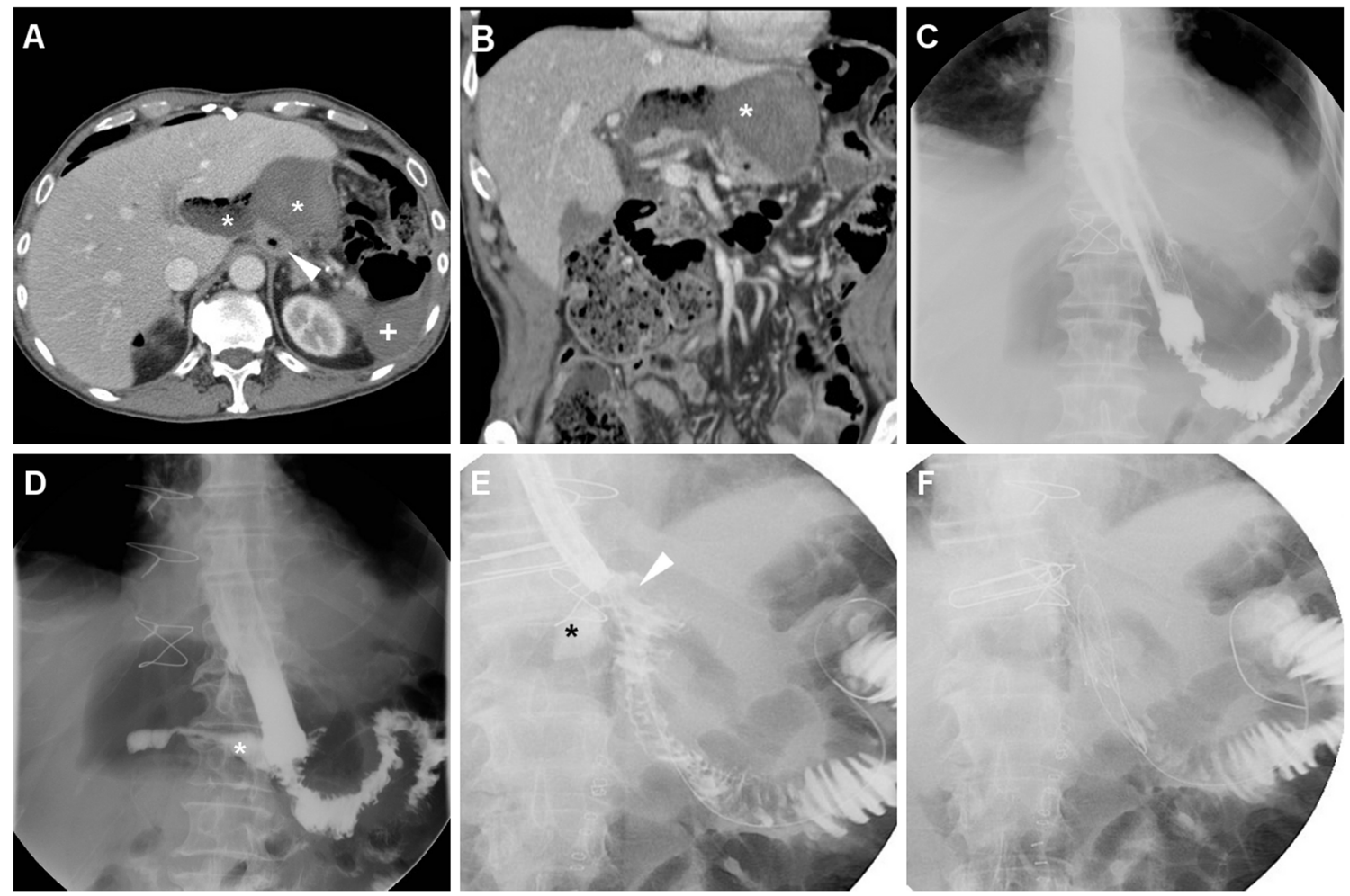

Fig. 11 A 68-year-old male with recurrent gastric carcinoma on previous Billroth II gastrectomy for peptic ulcer was treated with total gastrectomy. Postoperative anemization required blood transfusions. On 12th POD, contrast-enhanced CT (A, B) showed persistent intraperitoneal air, a mixed attenuation collection (*) adjacent to the EJS (arrowheads), consistent with anastomotic dehiscence, a subacute blood collection $(+)$ in the site of splenectomy. After percutaneous drainage, AL was treated with positioning of a metallic stent $(\mathrm{C})$ through the EJS. Persistent leakage of oral iodinated contrast (*) at follow-up CF (D, E) led to removal of the stent and positioning of a self-expanding stent $(\mathrm{F})$ the surrounding extraluminal compartment. On CT images the EJS or GJS should be carefully scrutinized for focal discontinuity and mural thickening. Since anastomotic dehiscence leads to the extravasation of enteral material in the surgical site, the most common appearance includes variably sized airfluid collections, abscesses or inflammatory changes which abut the EJS or GJS (Figs. 11 and 12) and tend to extend into the lesser sac, left subphrenic, gastrohepatic and gastrosplenic spaces [10-12].

Alternatively, anastomotic leak may sometimes appear as generalized peritonitis with free fluid and intraperitoneal air (Fig. 10). Suspicious findings which support a diagnosis of visceral perforation over residual postoperative air in the peritoneal cavity include persistence or increase of pneumoperitoneum, particularly if abundant (over $20 \mathrm{~cm}^{3}$ ) and occupying a single compartment. It has been reported that persistent air after POD 5 associated with leukocytosis has $80 \%$ sensitivity for identifying patients requiring re-operation [26, 27].

Albeit plain CT without enteral CM is rapidly performed even in critically ill patients, according to some reports positive oral $\mathrm{CM}$ improves the $\mathrm{CT}$ performance: leakage is heralded by extraluminal CM collecting nearby the EJS or GJS, or dissecting in the postoperative neocompartment (Figs. 8 and 13) [10-12, 22, 23].

In the past, diagnosis of anastomotic dehiscence warranted immediate surgical re-exploration with toilette, closure of leakage or redo anastomosis, placement of drainage tubes and enteral feeding. More recently, in stable patients with contained leaks and no peritonitis, non-operative management is increasingly considered: endoscopic stenting (Fig. 11) is becoming the preferred solution, and other options include endoscopic clipping (Fig. 14) and percutaneous drainage of fluid collections. Reoperation is currently reserved for wide dehiscence, peritonitis or failure of nonoperative management [4, $12,23]$.

\section{Anastomotic fistulas}

Well-known to radiologists, fistulas represent abnormal communications between the stomach and other structures such as the skin, bronchial tree or pleural cavity, which allow passage of enteral fluids. After gastrectomy, subphrenic infection secondary to anastomotic leak may occasionally be complicated by formation of 

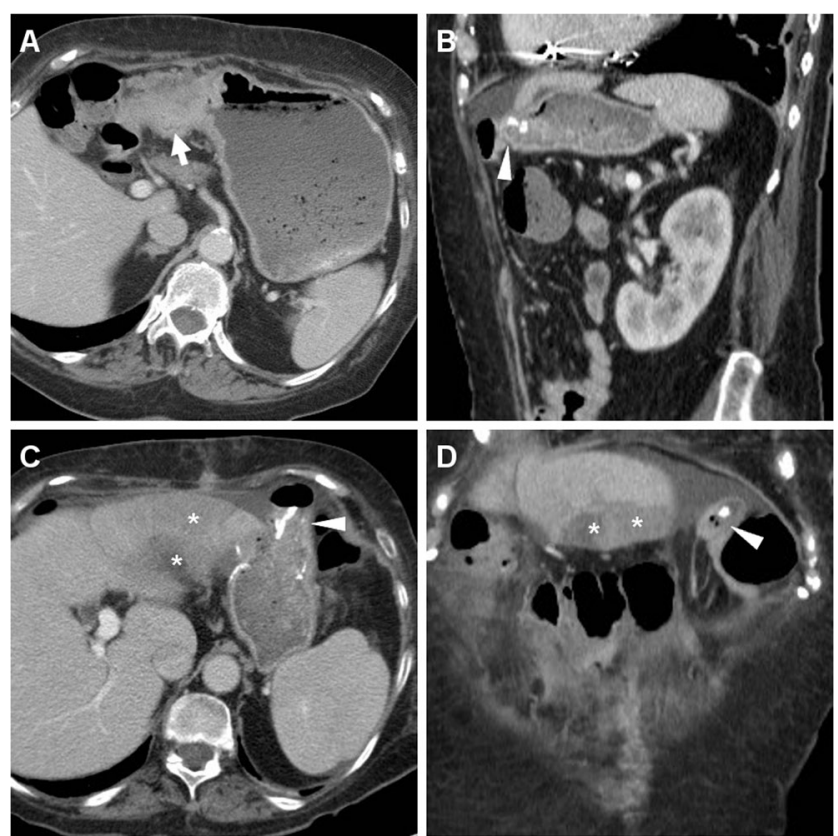

Fig. 12 An 81-year-old male with advanced, obstructing antral cancer (thick arrow) as preoperatively depicted by CT (A) underwent palliative partial gastrectomy with Roux-en-Y reconstruction. During prolonged hospitalization the patient experience anemization and failure to thrive. Early CF (not shown) showed slow transit through EJS. On 11th POD, contrast-enhanced CT (B-D) showed some ingested materials in the remnant stomach $(+)$, stapled EJS (arrowheads), some residual intraperitoneal air and fluid, inhomogeneous hypoattenuation of the 3rd liver segment consistent with parenchymal infection

gastro-cutaneous, gastro-bronchial or gastro-pleural (Fig. 14) fistulas, which represent rare occurrences described in sparse case reports. Albeit CF studies may show abnormal passage of $\mathrm{CM}$ through the fistula, CT more effectively depicts associated changes and consequences such as subphrenic abscess, pneumonia or empyema (Fig. 14) [10, 11].

\section{Anastomotic ulcers and strictures}

Anastomotic ulcers may develop after gastrectomy, but are unreliably depicted by imaging compared to endoscopy. Ulcers plus ischemia and scar formation may ultimately lead to the formation of an anastomotic stricture at the GJS (Fig. 7). Anastomotic strictures develop in up to $3-4.4 \%$ of operated patients, generally present weeks or months after surgery and manifest with nausea, vomiting, dysphagia or post-prandial pain [3].

\section{Duodenal stump complications}

\section{Afferent loop syndrome}

The uncommon afferent loop syndrome generally occurs after Billroth II reconstruction from obstruction of the afferent blind DS by adhesions, internal hernia, intraluminal blood or bezoar, anastomotic kinking, ulcer or stricture, and is heralded by fluid-filled duodenal dilatation (Fig. 15) [10, 11].

\section{Duodenal stump leakage}

A potentially worrisome (6-11.7\% mortality) complication of Billroth II reconstruction, DS leakage occurs after 3\% of partial gastrectomies and corresponds to the spread of bile from the blind-ending DS because of technical failure, ischemia or distal obstruction, causing local and ultimately peritoneal irritation [28]. Usual CT appearance includes a fluid collection abutting the stapled DS and extending to the right sub-hepatic or peripancreatic spaces (Figs. 6 and 9) [10, 11].

Currently, conservative treatment (parenteral nutrition, antibiotics, octreotide, suction drains and percutaneous drainage of abscesses) is successful in over $90 \%$ of cases;
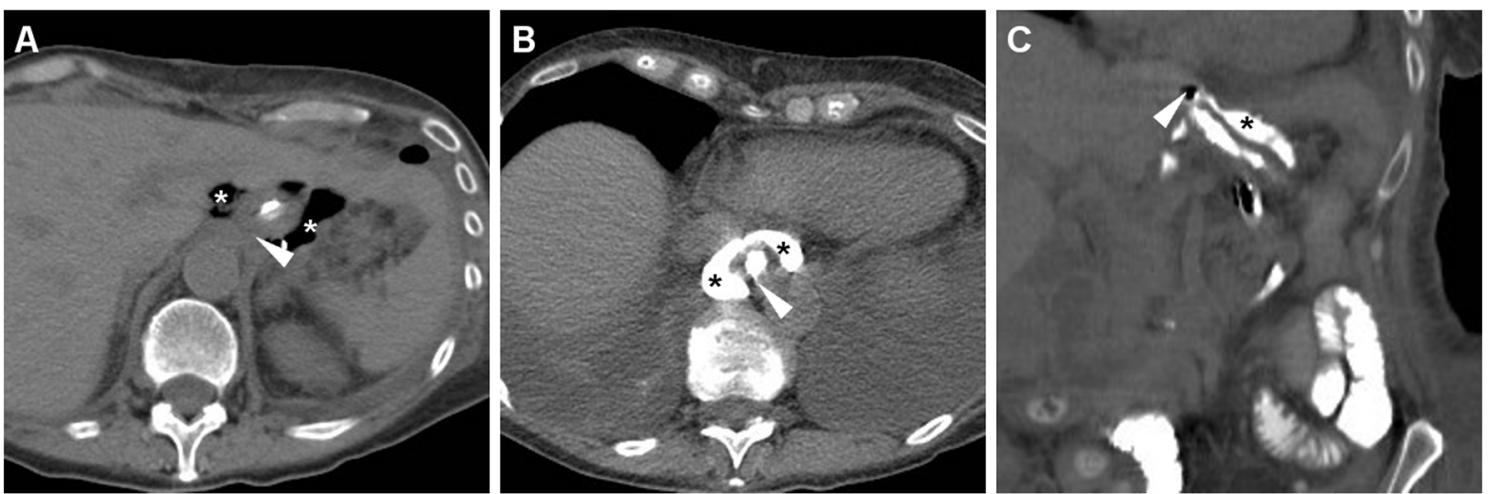

Fig. 13 In a 76-year-old woman with T4 N2 gastric cancer, three days after total gastrectomy unenhanced CT (A) showed minimal residual pneumoperitoneum and extensive air (*) surrounding the EJS (arrowheads). Repeated unenhanced CT with oral contrast administration (B, C) showed patent EJS (arrowheads) and confirmed circumferential anastomotic leakage $(*)$, which was initially managed conservatively but ultimately required reintervention with redo anastomosis six days later because of failed clinical improvement and progressive anemization 

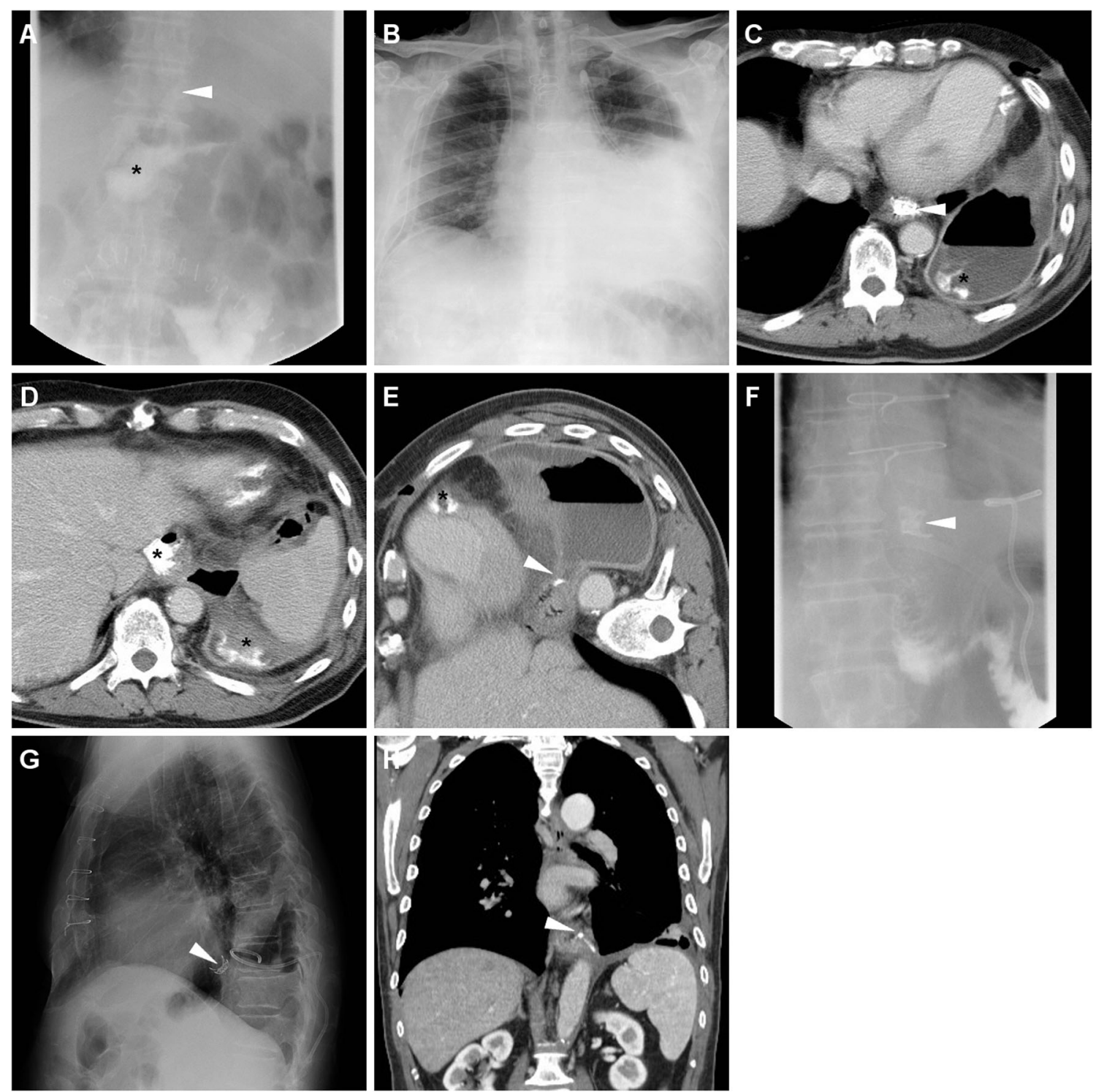

Fig. 14 A 59-year-old male with cardial carcinoma underwent total gastrectomy. Early postoperative CF (A) showed patent EJS (arrowhead) with perianastomotic contrast leakage (*) directed medially. Bilateral pleural drainages were positioned to relieve minimal right pneumothorax and left hydropneumothorax (B). On 10th POD contrastenhanced CT with oral contrast $(\mathrm{C}, \mathrm{D})$ confirmed ample left-sided hydropneumothorax with enhancing pleural surface. Extraluminal

contrast leakage $(*)$ was seen both medially to the EJS (arrowheads) and dependent in the left pleural empyema. Repeated CT obtained in the right lateral decubitus position (E) before CT-guided pleural drainage confirmed anastomotic fistula from the EJS (arrowhead) to the hydropneumothorax, which was treated by endoscopic clipping (arrowhead in F); note pleural pigtail drainage reoperation is reserved for septic patients or failure of conservative approach $[4,28]$.

\section{Vascular complications}

\section{Haemorrhage}

Blood loss after gastrectomy may represent a lifethreatening complication and generally results from inadequate haemostasis, particularly ligation of small feeding vessels. As it occurs at a staple line or anastomosis, early postoperative haemorrhage is most usually intraluminal rather than in the surgical site or peritoneal cavity. Conversely, delayed bleeding is mostly due to marginal anastomotic ulcers. CT findings include intraluminal high-attenuation blood or clots in the bowel (Fig. 15), sometimes extravascular CM "blush" corresponding to active bleeding. The latter sign and endoscopically occult haemorrhage represent indications for 

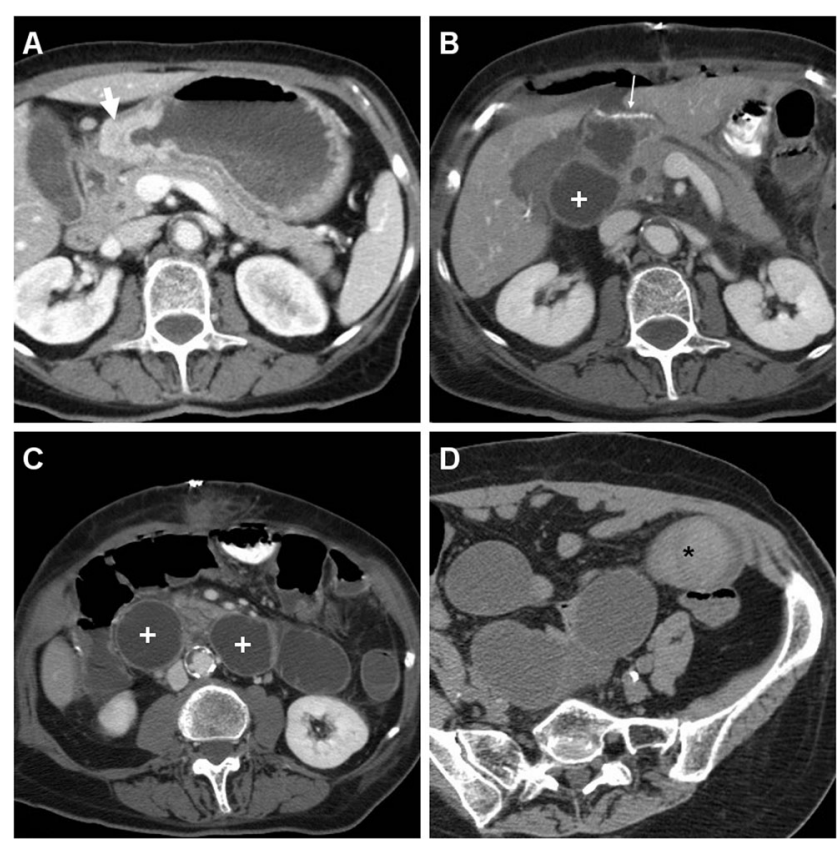

Fig. 15 A 72-year-old female with distal gastric carcinoma (thick arrow) as preoperatively depicted by CT (A) underwent total gastrectomy. On 6th POD the patient suffered from persistent abdominal pain and anemization. Postoperative CT (B-D) showed dilated fluid-filled duodenum (+) up to stapled DS, consistent with afferent limb syndrome, secondary to a large blood clot in the jejunum which was best seen as a hyperattenuating intraluminal mass $(*)$ on precontrast (D) scans. Surgical reintervention was required to relieve obstruction

transarterial embolisation, particularly in patients unfit for surgical reintervention [4, 29].

\section{Pancreatic fistula and pancreatitis}

Occurring after $7.6 \%$ of all gastrectomies, postoperative pancreatic fistula corresponds to leaking pancreatic secretions and develops secondary to resection or injury to the pancreatic capsule. Fatty pancreas and pancreas divisum are the key risk factors. The clinical and laboratory diagnosis requires any measurable output from peripancreatic drainage on or after POD 3 with amylase content over three times the serum amylase; alternatively pancreatic fistula is diagnosed at percutaneous drainage or surgical reintervention $[4,30]$.

At CT, the usual appearance is a focal fluid collection in the surgical bed, particularly in the lesser sac or adjacent to the pancreas (Fig. 16). Currently, postoperative pancreatic fistula is effectively managed nonsurgically until closure with parenteral nutrition and percutaneous drainage of dominant collections [31-33].

Alternatively, acute postoperative pancreatitis may develop in the gland remnant after partial pancreatectomy, and appears as segmental or diffuse enlargement with
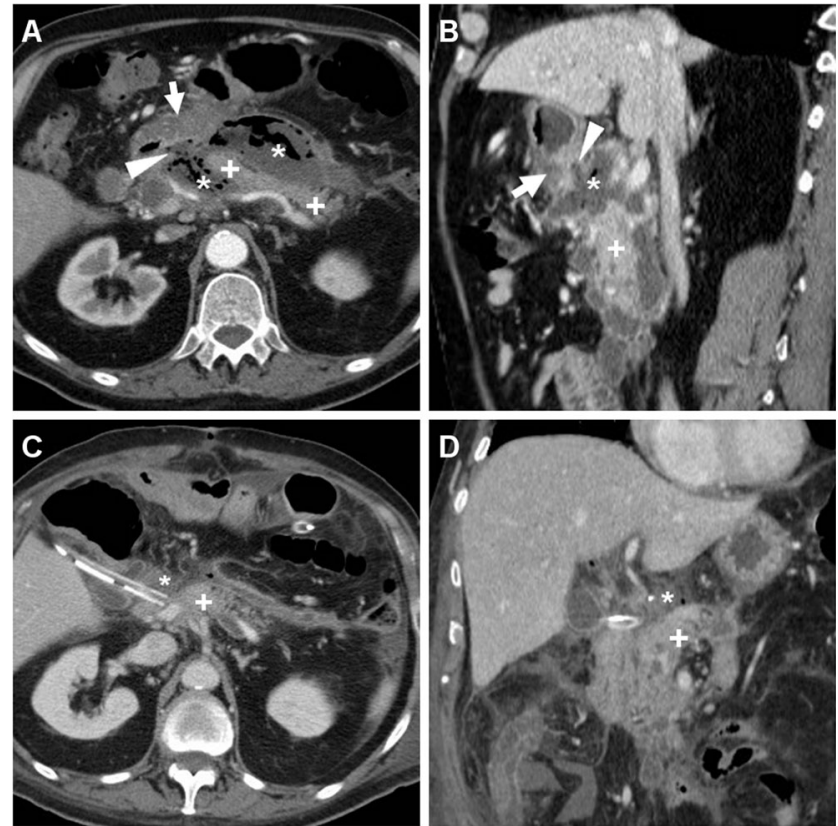

Fig. 16 An 84-year-old male with lesser sac and pancreatic abscess $(*$ in A, B) from retroperitoneal perforation of pyloric peptic ulcer (arrowheads) underwent emergency surgery, including subtotal gastrectomy with Billroth II reconstruction. Note pancreatic parenchyma $(+)$, oedematous thickening of pylorus submucosa (thick arrows). On 7th POD surgical revision was required due to DS biliary leakage, with positioning of Kehr tube. Subsequently, on 20th POD follow-up CT (C, D) showed minimal fluid adjacent to the drainage tube, consistent with laboratory diagnosis of postoperative pancreatic fistula

peripancreatic inflammatory changes and fluid collections extending to the retroperitoneal fasciae and the anterior pararenal space [4].

\section{Conclusion}

Albeit CF may still be used as first-line imaging, multidetector $\mathrm{CT}$ after radical gastrectomy increasingly allows comprehensive visualization of the operated abdominal compartment and provides a consistent basis for correct choice between conservative, interventional or surgical treatment. In our experience, early post-gastrectomy $\mathrm{CT}$ benefits from oral $\mathrm{CM}$ administration if permitted by the patient's conditions and cooperation. Understanding the surgically altered anatomy and knowledge of expected postoperative appearances is crucial to correctly recognizing complications.

Open Access This article is distributed under the terms of the Creative Commons Attribution 4.0 International License (http:// creativecommons.org/licenses/by/4.0/), which permits unrestricted use, distribution, and reproduction in any medium, provided you give appropriate credit to the original author(s) and the source, provide a link to the Creative Commons license, and indicate if changes were made. 


\section{References}

1. Smyth EC, Verheji M, Allum WH et al (2016) Gastric cancer: ESMO clinical practice guidelines for diagnosis, treatment and follow-up. Ann Oncol 27(Suppl 5):v38-v49

2. Deguchi Y, Fukagawa T, Morita S et al (2012) Identification of risk factors for esophagojejunal anastomotic leakage after gastric surgery. World J Surg 36:1617-1622

3. Inokuchi M, Otsuki S, Fujimori Y et al (2015) Systematic review of anastomotic complications of esophagojejunostomy after laparoscopic total gastrectomy. World J Gastroenterol 21:9656-9665

4. Weledji EP, Verla V (2016) Failure to rescue patients from early critical complications of oesophagogastric cancer surgery. Ann Med Surg (Lond) 7:34-41

5. Xiong JJ, Altaf K, Javed MA et al (2013) Roux-en-Y versus Billroth I reconstruction after distal gastrectomy for gastric cancer: a meta-analysis. World J Gastroenterol 19:1124-1134

6. Zilberstein B, Abbud Ferreira J, Cecconello I (2011) Management or postoperative complications in gastric cancer. Minerva Gastroenterol Dietol 57:69-74

7. Gaetke-Udager K, Wasnik AP, Kaza RK et al (2014) A guide to imaging in bariatric surgery. Emerg Radiol 21:309-319

8. Herron D, Roohipour R (2012) Complications of Roux-en-Y gastric bypass and sleeve gastrectomy. Abdom Imaging 37:712-718

9. Levine MS, Carucci LR (2014) Imaging of bariatric surgery: normal anatomy and postoperative complications. Radiology 270: 327-341

10. Kim KA, Park CM, Park SW et al (2002) CT findings in the abdomen and pelvis after gastric carcinoma resection. AJR Am J Roentgenol 179:1037-1041

11. Kim KW, Choi BI, Han JK et al (2002) Postoperative anatomic and pathologic findings at CT following gastrectomy. Radiographics 22:323-336

12. Kim TH, Kim JH, Shin CI et al (2015) CT findings suggesting anastomotic leak and predicting the recovery period following gastric surgery. Eur Radiol 25:1958-1966

13. Terrone DG, Lepanto L, Billiard JS et al (2011) A primer to common major gastrointestinal post-surgical anatomy on CT-a pictorial review. Insights Imaging 2:631-638

14. Straatman J, van der Wielen N, Cuesta MA et al (2016) Minimally invasive versus open total gastrectomy for gastric cancer: a systematic revie and meta-analysis of short-term outcomes and completeness of resection. World J Surg 40:148-157

15. Liang Y, Li G, Chen P et al (2011) Laparoscopic versus open gastrectomy for early distal gastric cancer: a meta-analysis. ANZ J Surg 81:673-680

16. Espat NJ, Karpeh M (1998) Reconstruction following total gastrectomy: a review and summary of the randomized prospective clinical trials. Surg Oncol 7:65-69
17. Kawamura Y, Satoh S, Umeki Y et al (2016) Evaluation of the recurrence pattern of gastric cancer after laparoscopic gastrectomy with D2 lymphadenectomy. SpringerPlus 5:821

18. Jiang L, Yang KH, Chen Yet al (2014) Systematic review and metaanalysis of the effectiveness and safety of extended lymphadenectomy in patients with resectable gastric cancer. Br J Surg 101:595604

19. Inokuchi M, Kojima K, Kato K et al (2014) Risk factors for postoperative pulmonary complications after gastrectomy for gastric cancer. Surg Infect (Larchmt) 15:314-21

20. Etoh T, Inomata M, Shiraishi $\mathrm{N}$ et al (2010) Revisional surgery after gastrectomy for gastric cancer: review of the literature. Surg Laparosc Endosc Percutan Tech 20:332-337

21. Adamina M, Steffen T, Tarantino I et al (2015) Meta-analysis of the predictive value of $\mathrm{C}$-reactive protein for infectious complications in abdominal surgery. Br J Surg 102:590-598

22. Hogan BA, Winter DC, Broe D et al (2008) Prospective trial comparing contrast swallow, computed tomography and endoscopy to identify anastomotic leak following oesophagogastric surgery. Surg Endosc 22:767-771

23. Struecker B, Chopra S, Heilmann AC et al (2017) Routine radiologic contrast agent examination after gastrectomy for gastric cancer is not useful. J Gastrointest Surg 21:801-806

24. Federle MP, Jaffe TA, Davis PL et al (2017) Contrast media for fluoroscopic examinations of the GI and GU tracts: current challenges and recommendations. Abdom Radiol (NY) 42:90-100

25. European Society of Urogenital Radiology (2016) ESUR guidelines on contrast media 9.0. Available at: www.esur.org/guidelines.

26. Hindman NM, Kang S, Parikh MS (2014) Common postoperative findings unique to laparoscopic surgery. Radiographics 34:119-138

27. Malgras B, Place V, Dohan A et al (2017) Natural history of pneumoperitoneum after laparotomy: findings on multidetector-row computed tomography. World J Surg 41:56-63

28. Aurello P, Sirimarco D, Magistri P et al (2015) Management of duodenal stump fistula after gastrectomy for gastric cancer: systematic review. World J Gastroenterol 21:7571-7576

29. Kim KH, Kim MC, Jung GJ et al (2012) Endoscopic treatment and risk factors of postoperative anastomotic bleeding after gastrectomy for gastric cancer. Int J Surg 10:593-597

30. Sato Y, Inokuchi M, Otsuki S et al (2017) Risk factor of pancreatic fistula after radical gastrectomy in the context of fatty pancreas. Dig Surg. doi:10.1159/000455332

31. Raman SP, Horton KM, Cameron JL et al (2013) CT after pancreaticoduodenectomy: spectrum of normal findings and complications. AJR Am J Roentgenol 201:2-13

32. Smith SL, Hampson F, Duxbury M et al (2008) Computed tomography after radical pancreaticoduodenectomy (Whipple's procedure). Clin Radiol 63:921-928

33. McEvoy SH, Lavelle LP, Hoare SM et al (2014) Pancreaticoduodenectomy: expected post-operative anatomy and complications. Br J Radiol 87:20140050 